\title{
The Applicability of Gd-DTPA Magnetic Resonance Imaging Contrast Agent for the Evaluation of Blood Compartment Flow Distribution in Hollow Fiber Hemodialyzers
}

\author{
Bimali Sanjeevani Weerakoon ${ }^{1,2 *}$, Toshiaki Osuga ${ }^{3}$ \\ ${ }^{1}$ Graduate School of Advanced Integration Science, Chiba University, Chiba, Japan \\ ${ }^{2}$ Department of Radiography/Radiotherapy, Faculty of Allied Health Sciences, University of Peradeniya, \\ Peradeniya, Sri Lanka \\ ${ }^{3}$ Center for Frontier Medical Engineering, Chiba University, Chiba, Japan

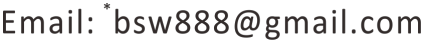

Received 25 September 2015; accepted 15 November 2015; published 18 November 2015

Copyright (C) 2015 by authors and Scientific Research Publishing Inc.

This work is licensed under the Creative Commons Attribution International License (CC BY). http://creativecommons.org/licenses/by/4.0/

(c) (i) Open Access

\begin{abstract}
Observation of flow distribution pattern in the hemodialyzers is significant as it is a valuable indication of the performance of these modules. Therefore, in this study, a feasible non-destructive Magnetic Resonance Imaging (MRI) technique is proposed to characterize the flow distribution in the blood compartment of hemodialyzers using Gd-DTPA MRI contrast agent. The distribution of flow is qualitatively observed in two commercial clinical dialyzers through an in-vitro experiment. The contrast enhanced T1 weighted images are acquired along the dialyzer length using Spin Echo (SE) pulse sequence after an injection of $0.5 \mathrm{mmol} / \mathrm{L}$ Gd-DTPA solution into the blood compartment. Although relatively uniform flow distribution pattern over the spatial volume of transverse images, close the dialyzer inlet is observed, the heterogeneity of flow distribution can be identified towards the blood outlet port. Furthermore, the signal intensity profiles formed by the injected Gd-DTPA are gradually decreased towards the outlet port. These results of the study suggest that although no advanced techniques and protocols available, MRI and Gd-DTPA contrast agent can be utilized to characterize the flow distribution within a dialyzer qualitatively.
\end{abstract}

\section{Keywords}

Hemodialyzer, Magnetic Resonance Imaging, Gd-DTPA Contrast Agent

\footnotetext{
${ }^{*}$ Corresponding author.
}

How to cite this paper: Weerakoon, B.S. and Osuga, T. (2015) The Applicability of Gd-DTPA Magnetic Resonance Imaging Contrast Agent for the Evaluation of Blood Compartment Flow Distribution in Hollow Fiber Hemodialyzers. J. Biomedical Science and Engineering, 8, 789-796. http://dx.doi.org/10.4236/jbise.2015.811075 


\section{Introduction}

An artificial kidney or hollow fiber hemodialyzer is the key functional unit in the hemodialysis therapy, which is designed to provide controllable transfer of uremic solutes and excesses plasma water from the blood of renal failure patients. Although there are several dialyzer configurations, the hollow fiber model is the most effective for blood filtration due to high surface area per unit blood volume. The blood filtration and mass transfer efficiency of the dialysis operation are affected by the geometrical and membrane physio-chemical characteristics of the hemodialyzers [1].

According to the various types of patients, there are different sizes of hemodialyzers available ranging from 2 - 5 $\mathrm{cm}$ in diameter and $15-30 \mathrm{~cm}$ in length. A typical hemodialyzer is enclosed in an acrylic, rigid, rounded shell, which houses a bundle of 10,000 - 12,000 extremely fine semi-permeable hollow fibers. These fibers have an approximately inner diameter of $200-210 \mu \mathrm{m}$ and a wall thickness of $15-50 \mu \mathrm{m}$ [1]-[3]. A tube sheet forms at the ends of potted fibers which separate the dialyzer into two regions of shell space and lumen space. The inside or the lumen of a hollow fiber is known as the blood compartment while the shell or outside of the lumen is known as the dialysate compartment. During the dialysis operation, blood is flowing in the opposite direction to the dialysate flow path within the hollow fibers [1] [3]. This counter-current flow is useful to maintain the concentration gradient across the membrane and removes the waste products from the blood. During the dialysis treatment, diffusion process caused by the concentration gradient of the fiber membrane is utilized to eliminate the smaller molecular weight uremic solutes such as urea and creatinine. Larger molecular weight solutes and excess plasma water are eliminated by convective transport through the membrane [1] [4]. In the clinical setting, typical blood flow rates and dialysis flow rates are maintained between $200-400 \mathrm{~mL} / \mathrm{min}$ and $500-800$ $\mathrm{mL} / \mathrm{min}$, respectively [1] [2].

In addition to the above structural properties and module design of hemodialyzers, distributions of flow within the blood and dialysate compartments are significant parameters of its performance in mass transfer efficiency [5] [6]. An efficient clearance of low molecular weight waste solutes from the blood is the main purpose of hemodialyzers and this process significantly depends on the optimization of diffusion between blood and dialysate solutions. The presence of uniform counter-current flow distributions in both compartments aid to maximize the mass transfer efficiency through the diffusion process [6]. Therefore, a mismatch between blood and dialysate flow distribution being impaired the diffusion process may cause the loss of uremic solute removal efficiency [1] [6].

Various efforts have been undertaken to develop theoretical models for predicting the effect of flow mal-distributions on mass transfer efficiency of a hemodialyzer [5] [7]. However, not like the theoretical models, the experimental knowledge of the flow distribution in hollow fiber hemodialyzer can provide significant informatio $\mathrm{n}$ on the actual fluid transportation phenomena. But the flow distribution measurements in these modules are challenging because of the complicated flow path in both directions with a smaller inner diameter of the fibers [1]. Due to this structural complexity, a reliable experimental approach for measuring the flow distribution within the dialyzers is limited. In spite of that, higher demanding nature of the efficiency have focused to improve the design of each component of the hemodialyzers [6]. Therefore, several different approaches for determining the flow distribution within the hollow fiber hemodialyzers have been attempted.

Park et al. [8] investigated the residence time distributions using tracer analysis to approximate the flow within the hollow fiber hemodialyzers. Using a related approach, Takesawa et al. [9] visualized the flow in the blood compartment using a radio-opaque dye and X-ray computed tomography. More quantitative non-destructive validated determinations of the flow distribution within the hollow fiber hemodialyzers have recently been attempted using Magnetic Resonance Imaging techniques. Flow distributions were evaluated using a Time-of Flight (TOF) approach [10] and alternative methods using velocity encoding with flow compensation [1] [11]. A more advanced method of MR hybrid multiple overlapping thin slab three-dimensional acquisition technique also applied to assess the bi-directional flow distribution [12].

The rapid expansion of using medical devices in the clinical health setting coupled with high efficiency has created a demand for evaluation of the functional efficacy of these hemodialyzers furthermore. Therefore, in this study, a feasible non-destructive Magnetic Resonance Imaging technique was proposed to characterize the flow distribution in the blood compartment of hemodialyzers using Gd-DTPA Magnetic Resonance Imaging (MRI) contrast agent. 


\section{Methods and Materials}

\subsection{Artificial Kidney Dialyzer}

In this study, two types of commercially available clinical hollow fiber hemodialyzers were utilized for in-vitro qualitative experimental observations. Characteristics of these hemodialyzers relevant to this study are shown in Table 1. Before starting the each experiment, the hemodialyzer was primed for approximately 2 hours, according to the procedure outlined in the manufacturer's direction sheet. This priming procedure helps to remove any surfactants and air bubbles that may have been present inside the hemodialyzer when it was first filled with the saline. Moreover, in this experiment, the blood and dialysate sides were considered as two independent domains [4]. Therefore, after the priming process with the saline, the dialysate ports were hermetically sealed throughout the experiment. The blood compartment with saline (as the compartmental fluid) was utilized for the experiment.

\subsection{Contrast Agent Preparation and Administration}

Gd-DTPA (Gadolinium-Diethylene Triamine Pentaacetic Acid/Magnevist-Schering, Germany) paramagnetic MRI contrast agent was applied to characterize and delineation of the blood side flow distribution in hemodialyzers. Therefore, the molecular weight of $743 \mathrm{~g} / \mathrm{mol} \mathrm{Gd-DTPA}$ was diluted with distilled water to prepare a concentration of $0.5 \mathrm{mmol} / \mathrm{L}$ aqueous solution. This selected concentration was decided after reviewing the previous studies [13]. After the flow had reached a steady state (circulating flow rate was $200 \mathrm{ml} / \mathrm{min}$ ) a bolus volume of Gd-DTPA solution was administered to the flow system manually using a syringe via a T-junction valve placed near the inlet port of the vertically placed dialyzer (from its bottom). Three dialyzers with each 50 $\mathrm{ml}$ and $25 \mathrm{ml}$ of contrast injection and without contrast injection (as a control) were utilized from the same manufacturer to make the comparison of contrast invasion pattern and distribution in one experiment. According to the factor of blood side capacity $(\mathrm{ml})$ for the hemodialyzers given by the manufacturers, the common injected contrast volumes of $25 \mathrm{ml}$ and $50 \mathrm{ml}$ were decided. The vortex generation was not expected in the study as the contrast administration was maintained at $200 \mathrm{ml} / \mathrm{min}$ input flow rate $(25 \mathrm{ml} / 7.5 \mathrm{~s}$ and $50 \mathrm{ml} / 15 \mathrm{~s})$, which is the rate utilized in actual clinical dialysis operations [1] [2].

\subsection{Magnetic Resonance Imaging and Image Acquisition}

After the contrast has been administered, the motion and the distribution of Gd-DTPA solution along the axial length of the hemodialyzer were observed with the acquisition of MR images. 0.3 Tesla (T) Hitachi AIRIS Vento (Hitachi Medical Corporation, Tokyo, Japan) the whole body open clinical MR scanner along with a birdcage head coil was used for image acquisition. To obtain the MR images, three dialyzers were mounted horizontally within the head coil with their longitudinal axis aligned with the z-gradient direction of the scanner. After this setting was inside the bore of the MR machine, T1 weighted images were acquired by applying the two-dimensional (2D) Spin Echo (SE) pulse sequence with the repetition time (TR) and echo time (TE) of 400 $\mathrm{ms}$ and $15 \mathrm{~ms}$, respectively. The flip angle was set to 90 degrees and the acquisition matrix size was set at $512 \times$ 512 with the field of view (FOV) of $220 \mathrm{~mm}^{2}$. Because of the sensitivity limitations of the head coil, this selected area only covered about $3 / 4$ from the total dialyzer length.

The imaging protocol consisted of one localizer image followed by non-contrast and contrast enhanced imaging sequences. After acquisition of the localizer, the first part of the imaging procedure has taken place only with the saline solution in the blood compartment and without contrast injection. Sequentially, the second part of the imaging procedure was performed with the injection of contrast solution into the two dialyzers (with $25 \mathrm{ml}$ and $50 \mathrm{ml}$ of contrast injections). Immediately after the injections, MRI scanning has taken place and the total image acquisition time for one imaging sequence was six minutes. The flow circulation was terminated during the scan period to avoid the motion artifacts and to demonstrate the frontal zone of the injected contrast solution.

Table 1. Physical characteristics of hemodialyzers (given by the manufacturer).

\begin{tabular}{|c|c|c|c|}
\hline Type of the Dialyzer & Manufacturer & Effective Surface Area $\left(\mathrm{m}^{2}\right)$ & Blood Side Capacity (ml) \\
\hline FDY-180 GW & Nikkiso Tokyo, Japan. & 1.8 & 108 \\
\hline $\mathrm{NF}-1.8 \mathrm{H}$ & Toray Medical Tokyo, Japan. & 1.8 & 112 \\
\hline
\end{tabular}


To avoid misconceptions due to the cylindrical structure, the central vertical image and four (4) transverse images at different locations were obtained along the axial direction of the each hemodialyzer before and after contrast has been administered. The distance of each transverse cross section was set at $20 \mathrm{~mm}$ with the slice thickness of $5 \mathrm{~mm}$. The entry slice was positioned approximately $2 \mathrm{~cm}$ away at the starting point of the fiber bundle on the scanogram. This same technique with the same parameters was used for all two types of dialyzers to evaluate the flow distribution in the blood compartment. Each experiment was repeated once to confirm the reproducibility. The temperature of the liquids in the dialyzer was maintained at $25^{\circ} \mathrm{C}$ room temperature.

\subsection{Image Analysis}

The subsequent four transverse images and the central vertical image of each hemodialyzer were utilized for qualitative visual analysis. The localizer of each examination was used to identify the locations of the images and ImageJ (National Institutes of Health, USA) software was applied for processing the images. An additional image analysis was carried out utilizing Microsoft visual studio 2008 and Graph R software (version 1.67) which allows the generation of three-dimensional (3D) signal intensity plots with tracking of nano-meter scale movements inside the hemodialyzer.

\section{Results}

\subsection{Spatial Flow Distribution of the Contrast Solution}

Figure 1 demonstrates the comparison of representative threshold MRI images of the central vertical section and transverse sections of the same dialyzer after commencing the injection volumes of $25 \mathrm{ml}$ and $50 \mathrm{ml} \mathrm{Gd-DTPA}$ solution. The injected Gd-DTPA solution and the already present water are represented by black and white regions, respectively. Considering the position of the inlet port at the left end of the hemodialyzer, the motion of the injected Gd-DTPA solution towards outlet port can be shown in this figure. In Figures 1(a)-(d), sequential threshold MR images are displayed in order from the blood inlet to the outlet and it indicates the radial direction flow at various transverse planes of the hemodialyzers. This provides the comparison of the spatial distribution of injected Gd-DTPA solution over each transverse section corresponding to different positions of the central vertical images.

\subsection{Comparison of Non-Contrast and Contrast-Enhanced Signal Intensity Profiles}

Figure 2 indicates the representative central vertical images of the hemodialyzers. Figure 2(a), was taken before contrast injection (pre-contrast) and Figure 2(b) was taken sequentially after (25 $\mathrm{ml}$ and $50 \mathrm{ml}$ injections into two dialyzers) contrast injections (post-contrast). With respect to the above, example three-dimensional (3D)

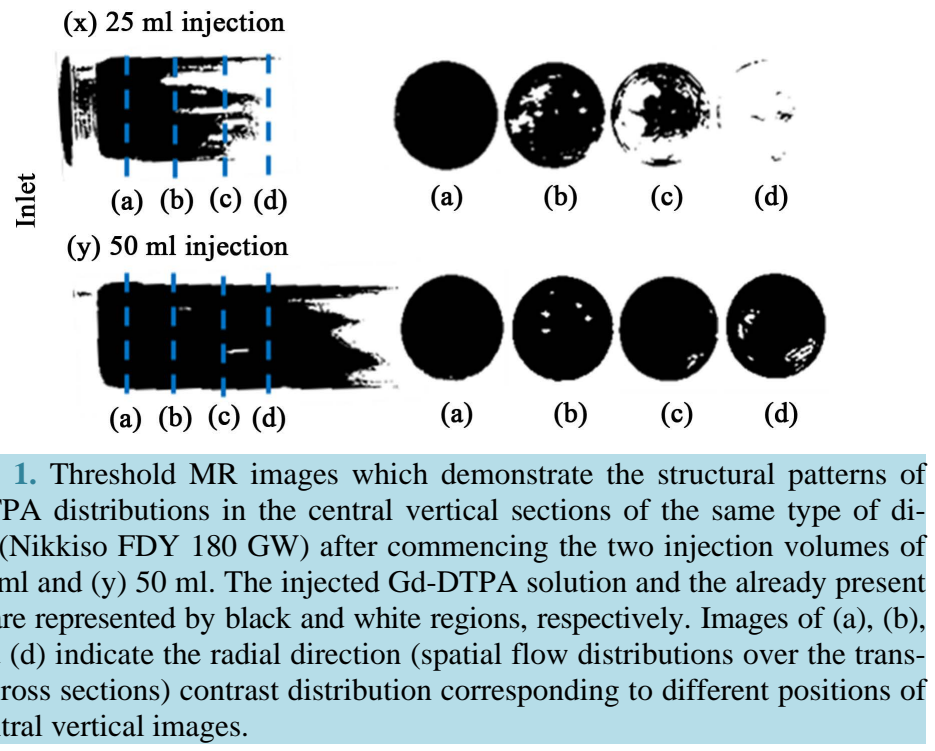

\footnotetext{
Figure 1. Threshold MR images which demonstrate the structural patterns of Gd-DTPA distributions in the central vertical sections of the same type of dialyzer (Nikkiso FDY $180 \mathrm{GW}$ ) after commencing the two injection volumes of (x) $25 \mathrm{ml}$ and (y) $50 \mathrm{ml}$. The injected Gd-DTPA solution and the already present water are represented by black and white regions, respectively. Images of (a), (b), (c) and (d) indicate the radial direction (spatial flow distributions over the transverse cross sections) contrast distribution corresponding to different positions of the central vertical images.
} 
signal intensity (SI) profiles which reflects the contrast flow distribution within the $\mathrm{x}, \mathrm{y}$ and $\mathrm{z}$ coordinates towards the outlet port in hemodialyzers are shown in Figure 2(c) and Figure 2(d). In the absence of contrast solution, there is no significant difference of the SI profiles between each three dialyzer and they indicate iso-intense SI profiles. The highest SI was observed at the inlet port and it gradually decreased towards the outlet port along the axial-direction of the hemodialyzer after contrast has administered. The hemodialyzers with 50 $\mathrm{ml}$ of contrast injection relatively appear as hyper-intense compare to the $25 \mathrm{ml}$ of injection (Figure 2(b) and Figure 2(d)).

\subsection{Invasion of Flow}

The invasion pattern of the injected Gd-DTPA solution in the hemodialyzers are indicated by Figure 3. This illustrates three example 3D SI plots describing spatial distribution and the invasion pattern at three transverse

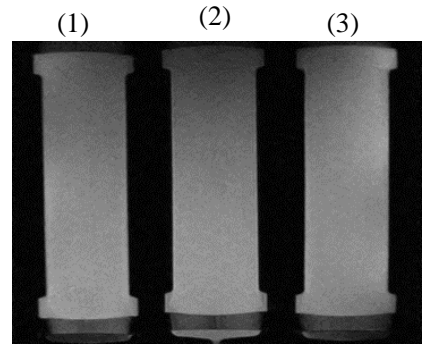

(a) Pre-contrast

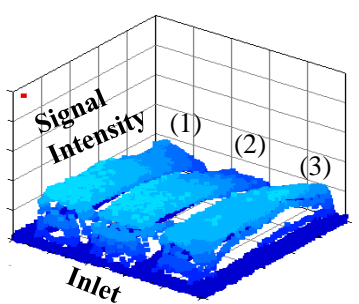

(c) Pre-contrast

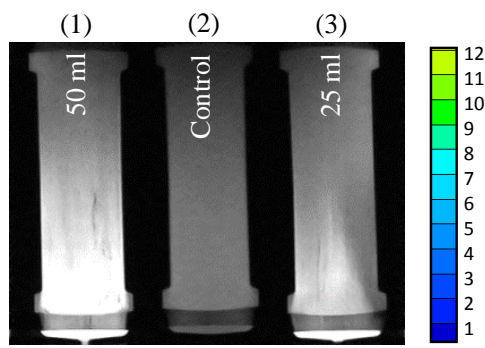

(b) Post-contrast

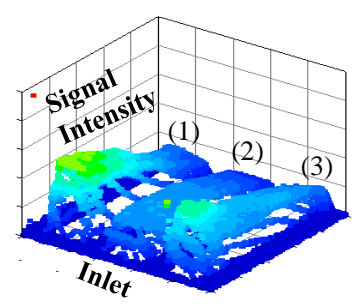

(d) Post-contrast

Figure 2. (a), (b) MR images and (c), (d) signal intensity profiles of central vertical section of the same type (TorayNF-1.8H) of hemodialyzers. (a), (c) Before contrast injections (precontrast) and (b), (d) after contrast injections (post-contrast). (1) $50 \mathrm{ml}$ contrast injection; (2) control or without contrast injection; and (3) $25 \mathrm{ml}$ contrast injection.

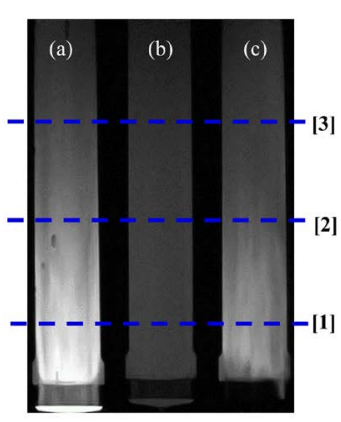

Inlet
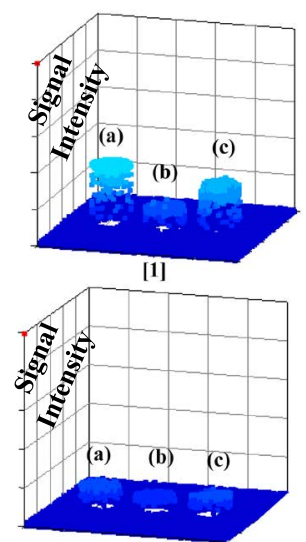

[3]

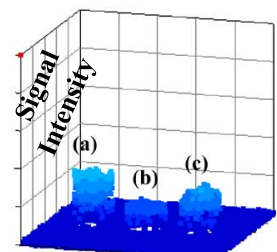

[2]
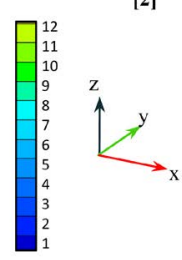

Figure 3. Comparison of the invasion pattern and the spatial distribution of Gd-DTPA solution at different levels of transverse sections in the same type (Nikkiso FDY 180GW) of hemodialyzers. (a) $50 \mathrm{ml} \mathrm{Gd-DTPA}$ injection; (b) without contrast injection and (c) 25 ml Gd-DTPA injection. 
imaging planes within the same type of hemodialyzers. It also allows for comparison the spatial distribution between each transverse imaging plane with different injection levels. Relatively hyper-intense, uniform spatial distribution can be observed in the cross sections close to the blood entrance.

\section{Discussion}

The experimental knowledge of the flow distribution in hollow fiber hemodialyzers plays a significant approach into the actual fluid transportation phenomena of these modules. The mass transfer efficiency through the diffusion process in the hemodialyzer is affected by the optimized flow distribution. However, there has been no exact validated methodology to evaluate the uniformity of blood compartment flow distribution in the hollow fiber hemodialyzers. Therefore, a preliminary feasible experiment was described here by using Gd-DTPA MRI contrast agent which can be applied to evaluate the blood compartment flow distribution in a clinical dialyzer. This proposed experiment based on the behavior of contrast agent distribution in the blood compartment and the utilization of MRI technique to visualize this dispersion. Therefore, flow distribution can be visualized here by means of a signal intensity distribution. Under the ideal uniform flow condition, blood should homogeneously distribute over the transverse spatial volume of the dialyzer [1]. The observation of flow within the both longitudinal and transverse directions was capable in this study.

The pre and post contrast imaging sequences were useful to compare the signal intensity distributions of dialyzers before and after contrast administration. Moreover, non-contrast imaging sequence also important to identify the effect of magnetic field in homogeneity for the imaging procedure. The dialyzer, which was used as the control in the post contrast imaging sequence provides a better understanding of the signal enhancement furthermore. The Spin Echo scan protocol, which was applied for image acquisition is not sensitive to the flow imaging. Hence the flow was terminated during the scan period to avoid the motion blur artifact. In previous studies [14], Gd-DTPA contrast solution was used as a tracer molecule for the observation of molecular transport in water using MRI. Therefore, in this experiment, the Gd-DTPA was used as the contrast agent to delineate the flow distribution. The Gd-DTPA also able to modify the longitudinal relaxation time of the water to enhance the visibility of MR images [14]. Because of this, the application of Gd-DTPA has the capability to precisely delineate the actual flow distribution.

Furthermore, due to the reproducible purpose and easy handling (handling of the blood samples requires a special training on aseptic techniques); all experiments in this study were performed in the presence of saline in the blood compartment. Therefore in this experiment, it was based on the assumption that no effects of human blood cells and proteins on the properties of fluid transport in these hemodialyzers. In general, because of the loss of plasma water, the viscosity of human blood is expected to increase as it travels along the length of a hemodialyzer [15]. Because of this, further verification is required with the blood to identify the effect of hydraulic properties towards the flow distribution.

The visible structural patterns formed by the injected Gd-DTPA signal intensity profiles in Figure 1, were demonstrated that close to the inlet port, the contrast solution was relatively homogeneously distributed over the entire transverse spatial volume of the dialyzer. But with the presence of unequally distributed Gd-DTPA solution over the transverse sections, the visible heterogeneity was demonstrated in the structural pattern of the contrast solution towards the outlet port. In addition to that, the shape of the frontal zone of injected Gd-DTPA solution has become fairly parabolic appearance at the distal ends. This observation was partially in agreement with some of the previous studies [11] [16] [17] which were evaluated the flow distribution with different imaging techniques. With the application of MR Fourier velocity imaging [16] and CT multiple segmentation method [17], the previous investigators were reported that the shape of the blood side flow distribution had a parabolic appearance.

The region of the blood inlet port where the injection was administered exhibited high signal intensity levels. However, the mixing process of Gd-DTPA with already present blood compartment fluid caused to decrease the signal intensity levels gradually towards the outlet port in contrast-enhanced dialyzers as demonstrated in Figure 2. In addition to that, it is worthwhile to point out that the flow distributions were reported here with the assumption of uniformity of the fiber densities over each transverse section. This assumption may not be true as always due to the practical conditions. Therefore, some of the variations reported in flow distributions can be due to the fiber density variabilities or inadequate priming conditions of the experiment. The observations of spatial distribution and the invasion pattern of the injected Gd-DTPA solution in Figure 3 was demonstrated 
that the progression of the flow was remarkably homogeneous and discrepancy between the flow distribution of central and peripheral fibers were virtually absent close to the inlet port. The studies done by Hardy et al. [11] and Zhang et al. [12] with the use of MR Fourier velocity imaging were also reported relatively uniform flow distribution within the hollow fibers. Another study [18] with the use of contrastographic Computed Tomography examination had similar observation and concluded the good design in the blood flow path geometry with the application of human blood.

$$
U_{\text {ave }}=\frac{Q}{\pi r^{2}}
$$

As expressed in Equation (1), in the dialysis operation, the approximately estimated averaged blood compartment flow velocity $\left(U_{\text {ave }}\right)$ through a hollow fiber is $5.3 \mathrm{~mm} / \mathrm{Sec}\left(200 \mathrm{~mL} / \mathrm{min} / \pi \times(2 \mathrm{~cm})^{2} \times 0.5\right)$ at the flow rate $(Q)$ of $200 \mathrm{ml} / \mathrm{min}$ (Fiber bundle radius $(r) \approx 2 \mathrm{~cm}$, Hollow fiberdensity $(\mathrm{fb}) \approx 0.5$ ). Therefore, the averaged time required to pass through the hollow fiber for blood compartment fluid is calculated to be 50 Sec (if the approximate length of the fiber bundle is considered as $25 \mathrm{~cm}$, the time required to pass the total length of fiber is $25 \mathrm{~cm} / 0.5 \mathrm{~cm} / \mathrm{Sec})$. At the same time, the time required to perform one MRI scan in this study was 6 mins.

$$
(\Delta d)^{2}=2 D t
$$

The displacement $\Delta d$ after starting molecular diffusion at time $t=0$ can be described from Equation (2) [19] where $D$ is the diffusion coefficient of the Gd-DTPA molecules in water (The diffusion coefficient of M.W. 743 Gd-DTPA at $25^{\circ} \mathrm{C}$ is assumed as $0.4 \times 10^{-9} \mathrm{~m}^{2} / \mathrm{Sec}$ ). Therefore, the averaged Brownian motion (from Equation (2)) of Gd-DTPA molecules for $50 \mathrm{Sec}$. and 6 min are approximately $200 \mu \mathrm{m}\left(d^{2}=2 \times 0.4 \times 10^{-9} \mathrm{~m}^{2} / \mathrm{Sec} \times 50\right.$ Sec) and $536 \mu \mathrm{m}\left(d^{2}=2 \times 0.4 \times 10^{-9} \mathrm{~m}^{2} / \mathrm{Sec} \times 360 \mathrm{Sec}\right)$ respectively, which are the scale order of the cross section of the hollow fiber. Therefore, the effect of the diffusion displacement of Gd-DTPA during the scan period was assumed to be negligible and signal enhanced region due to the motion of Gd-DTPA, expected to reflect the precise flow direction of injected contrast solution. Furthermore, because the contrast injection was done using the $200 \mathrm{ml} / \mathrm{min}$ input flow rate, the vortex generation in the blood compartment flow path was not expected here.

\section{Conclusion}

This paper has demonstrated a more feasible qualitative approach of MRI technique and Gd-DTPA contrast agent to observe the flow distribution in the blood compartment of a clinical artificial dialyzer. As such, this study is evaluated the behavior of contrast agent distribution within the blood compartment utilizing the MRI technique. Therefore, the reported flow distributions can be visualized here by means of the signal intensity distributions. Beside this, the technique is facilitated to evaluate only the uni-directional flow of these fiber modules. Moreover, because the blood is considered as a non-Newtonian fluid, multiple factors should evaluate, such as the effect of temperature and presence of hematocrits to predict the real blood flow distribution. Therefore, it is also important to highlight that further reviewing of this technique is essential, especially in the presence of hematocrits and under various operating conditions to improve the applicability of this method to the clinical settings.

\section{Acknowledgements}

The authors would like to highly appreciate and thankful for the support given by the Ministry of Education, Culture, Sports, Science, and Technology (MEXT: Japanese Government MONBUKAGAKUSHO scholarship program) in Japan.

\section{References}

[1] Poh, C.K., Hardy, P.A., Liao, Z., Huang, Z., Clark, W.R. and Gao, D. (2003) Effect of Flow Baffles on the Dialysate Flow Distribution of Hollow-Fiber Hemodialyzers: A Nonintrusive Experimental Study Using MRI. Journal of Biomechanical Engineering, 125, 481-489. http://dx.doi.org/10.1115/1.1590355

[2] Liao, Z., Poh, C.K., Huang, Z., Hardy, P.A., Clark, W.R. and Gao, D. (2003) A Numerical and Experimental Study of Mass Transfer in the Artificial Kidney. Journal of Biomechanical Engineering, 125, 472-480. http://dx.doi.org/10.1115/1.1589776 
[3] Osuga, T., Obata, T. and Ikehira, H. (2004) Proton Magnetic Resonance Imaging of Flow Motion of Heavy Water Injected into a Hollow Fiber Dialyzer Filled with Saline. Magnetic Resonance Imaging, 22, 413-416. http://dx.doi.org/10.1016/j.mri.2003.07.003

[4] Ding, W., Li, W., Sun, S., Zhou, X., Hardy, P.A., Ahmad, S. and Gao, D. (2015) Three-Dimensional Simulation of Mass Transfer in Artificial Kidneys. Artificial Organs, 39, E79-E89. http://dx.doi.org/10.1111/aor.12415

[5] Vander Velde, C. and Leonard, E.F. (1985) Theoretical Assessment of the Effect of Flow Mal-Distributions on the Mass Transfer Efficiency of Artificial Organs. Medical and Biological Engineering and Computing, 23, $224-229$. http://dx.doi.org/10.1007/BF02446862

[6] Ronco, C., Brendolan, A., Crepaldi, C., Rodighiero, M. and Scabardi, M. (2002) Blood and Dialysate Flow Distributions in Hollow-Fiber Hemodialyzers Analyzed by Computerized Helical Scanning Technique. Journal of the American Society of Nephrology, 13, S53-S61.

[7] Annan, K. (2012) Mathematical Modeling of the Dynamic Exchange of Solutes during Bicarbonate Dialysis. Mathematical and Computer Modelling, 55, 1691-1704. http://dx.doi.org/10.1016/j.mcm.2011.11.013

[8] Park, J.K. and Chang, H.N. (1986) Flow Distribution in the Fiber Lumen Side of a Hollow-Fiber Module. AIChE Journal, 32, 1937-1947. http://dx.doi.org/10.1002/aic.690321202

[9] Takesawa, S., Terasawa, M., Sakagami, M., Kobayashi, T., Hidai, H. and Sakai, K. (1988) Nondestructive Evaluation by X-Ray Computed Tomography of Dialysate Flow Patterns in Capillary Dialyzers. ASAIO Journal, 34, 794-799.

[10] Pangrle, B.J., Walsh, E.G., Moore, S. and DiBiasio, D. (1989) Investigation of Fluid Flow Patterns in a Hollow Fiber Module Using Magnetic Resonance Velocity Imaging. Biotechnology Techniques, 3, 67-72. http://dx.doi.org/10.1007/BF01876224

[11] Poh, C.K., Hardy, P.A., Liao, Z., Clark, W.R. and Gao, D. (2003) Nonintrusive Characterization of Fluid Transport Phenomena in Hollow-Fiber Membrane Modules Using MRI: An Innovative Experimental Approach. Membrane Science and Technology, 8, 89-122. http://dx.doi.org/10.1016/S0927-5193(03)80008-6

[12] Zhang, J., Parker, D.L. and Leypoldt, J.K. (1995) Flow Distributions in Hollow Fiber Hemodialyzers Using Magnetic Resonance Fourier Velocity Imaging. ASAIO Journal, 41, M678-M682. http://dx.doi.org/10.1097/00002480-199507000-00097

[13] Osuga, T., Obata, T. and Ikehira, H. (2004) Detection of Small Degree of Nonuniformity in Dialysate Flow in Hollow-Fiber Dialyzer Using Proton Magnetic Resonance Imaging. Magnetic Resonance Imaging, 22, 417-420. http://dx.doi.org/10.1016/j.mri.2003.08.008

[14] Gussoni, M., Greco, F., Vezzoli, A., Osuga, T. and Zetta, L. (2001) Magnetic Resonance Imaging of Molecular Transport in Living Morning Glory Stems. Magnetic Resonance Imaging, 19, 1311-1322. http://dx.doi.org/10.1016/S0730-725X(01)00468-4

[15] Ronco, C., Ghezzi, P.M., Metry, G., Spittle, M., Brendolan, A., Rodighiero, M.P., Milan, M., Zanella, M., Greca, G.L. and Levin, N.W. (2001) Effects of Hematocrit and Blood Flow Distribution on Solute Clearance in Hollow-Fiber Hemodialyzers. Nephron, 89, 243-250. http://dx.doi.org/10.1159/000046080

[16] Hardy, P.A., Poh, C.K., Liao, Z., Clark, W.R. and Gao, D. (2002) The Use of Magnetic Resonance Imaging to Measure the Local Ultrafiltration Rate in Hemodialyzers. Journal of Membrane Science, 204, 195-205. http://dx.doi.org/10.1016/S0376-7388(02)00038-8

[17] Lu, J. and Lu, W.Q. (2010) Blood Flow Velocity and Ultra-Filtration Velocity Measured by CT Imaging System inside a Densely Bundled Hollow Fiber Dialyzer. International Journal of Heat and Mass Transfer, 53, 1844-1850. http://dx.doi.org/10.1016/j.ijheatmasstransfer.2010.01.005

[18] Brendolan, A., Ronco, C., Ghezzi, P.M. and La Greca, G. (1999) Hydraulic and Flow Dynamic Characteristics of Vitamin E-Bonded Dialyzers. Contributions to Nephrology, 127, 79-88. http://dx.doi.org/10.1159/000059989

[19] Osuga, T. and Han, S. (2004) Proton Magnetic Resonance Imaging of Diffusion of High-and Low-Molecular-Weight Contrast Agents in Opaque Porous Media Saturated with Water. Magnetic Resonance Imaging, 22, 1039-1042. http://dx.doi.org/10.1016/j.mri.2003.07.004 